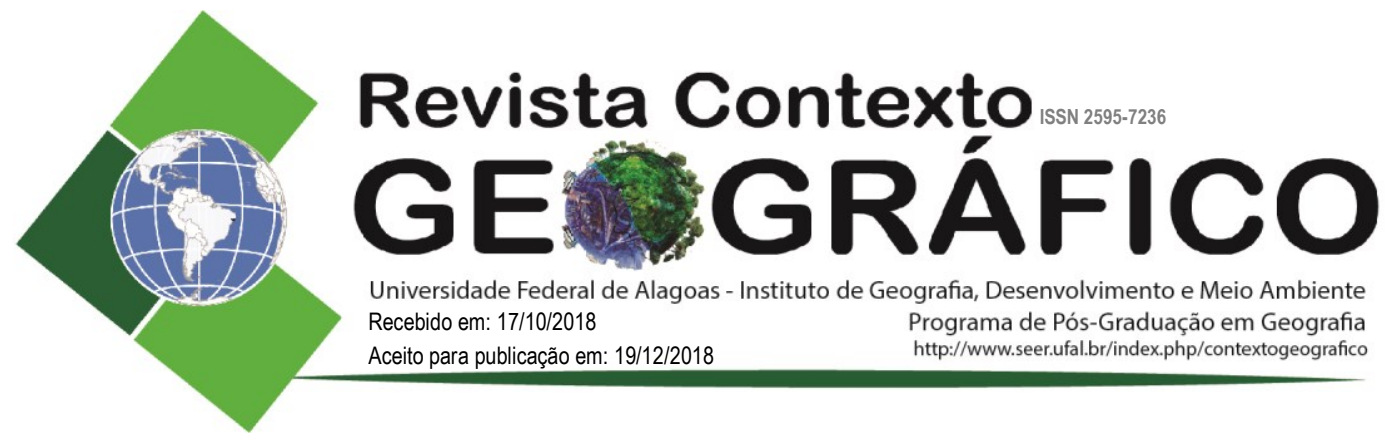

\title{
O USO DE PRODUTOS DE SENSORIAMENTO REMOTO EM ESTUDOS GEOMORFOLÓGICOS: UM OLHAR SOB OS SIMPÓSIOS NACIONAIS
}

\author{
Laís Susana de Souza Gois \\ Universidade Federal de Alagoas, Pós-Graduação em Geografia, Maceió, AL, Brasil \\ lais.gois@igdema.ufal.br
}

Jardel Estevam Barbosa dos Santos

Universidade Federal de Alagoas, Instituto de Geografia, Desenvolvimento e Meio Ambiente, Maceió, AL, Brasil

jardel.santos@igdema.ufal.br

Yan Carlos da Rocha Vasconcelos

Universidade Federal de Alagoas, Instituto de Geografia, Desenvolvimento e Meio Ambiente,

Maceió, AL, Brasil

yan.vasconcelos@igdema.ufal.br

\begin{abstract}
RESUMO - A geomorfologia é um ramo da ciência que se preocupa com a morfologia da Terra, porém para que se tenha um conhecimento de qualidade desse objeto de estudo é necessário o uso de ferramentas que aperfeiçoem as pesquisas. O sensoriamento remoto há algum tempo já vem sendo utilizado pelos profissionais da área e vem dando resultados satisfatórios aos pesquisadores à medida que as pesquisas vêm aumentando, seja para caracterizar, monitorar, avaliar e demarcar áreas. Com o intuito de avaliar alguns aspectos dessa produção, nesta pesquisa foram selecionados todos os trabalhos que mostram a aplicação do sensoriamento remoto nos estudos geomorfológicos no Simpósio Nacional de Sensoriamento Remoto (SBSR) nas edições de 2015 e 2017 e o Simpósio Nacional de Geomorfologia (SINAGEO) nas edições de 2016 e 2018. Foi feita a espacialização desses trabalhos de acordo com as regiões. Em seguida foram divididos em 4 linhas de pesquisa dentro da geomorfologia, e os trabalhos de cada linha foram divididos em subcategorias de objetivos. A partir dessas divisões foi possível perceber o uso de diferentes dados. Uso esse que pode ser limitado dentre outros fatores, pela escala e pelo custo, tanto dos dados quanto dos softwares.
\end{abstract}

Palavras-chave: Geomorfologia; SBSR; SINAGEO; Produção Acadêmica.

\section{THE STATE OF THE ART OF REMOTE SENSORING APPLIED TO GEOMORPHOLOGICAL STUDIES IN NATIONAL}

\begin{abstract}
Geomorphology is a branch of science that is concerned with the morphology of the Earth, but in order to have quality knowledge of this object of study it is necessary to use tools that optimize the research. Remote sensing has been used for some time by professionals in the field and has been providing satisfaction to researchers as research has been increasing, whether to characterize, monitor, evaluate and demarcate areas. In this research, we selected all the works that show the application of remote sensing in geomorphologic studies in the National Remote Sensing Symposium (SBSR) in the editions of 2015 and 2017 and the National Symposium on Geomorphology (SINAGEO) in the editions of 2016 and 2018. The works were spatialized according to the regions. They were then divided into 4 lines of research within the geomorphology, and the works of each line were divided into sub categories of objectives. From these divisions it was possible to perceive the use of different data. This use may be limited, among other factors, by the scale and cost of both data and software.
\end{abstract}

Keywords: Geomorphology; SBSR; SINAGEO; Academic Production. 


\title{
INTRODUÇÃO
}

Dentro da ciência geográfica, a Paisagem é um dos conceitos mais utilizados no ramo da Geografia Física. Corrêa (2005), explica que esta categoria é controlada pela geomorfologia e a geologia, pelas associações biogeográficas, ou mesmo pela predominância de determinadas associações de processos superficiais desencadeados ou não pela ação antrópica. Desta forma os estudos geomorfológicos tornam-se imprescindíveis na análise e aplicação deste conceito.

Nesse sentido, ao longo do tempo os pesquisadores vêm buscando maneiras de aperfeiçoar os estudos geomorfológicos, visto que em países como o Brasil a variação morfológica é elevada e carece de estudos detalhados. Sendo assim o sensoriamento remoto surge como uma opção bastante viável técnica e metodologicamente falando.

Silva e Santos (2012) apud Florenzano (2008), consideram que:

\begin{abstract}
A partir da utilização da fotografia, a geomorfologia passou a se beneficiar do sensoriamento remoto, por meio das quais passou a ser possível a obtenção de informações sobre o relevo com riqueza de detalhes, além de dados sobre o uso e cobertura da terra, dos sistemas hidrológicos, da estrutura geológica, entre outros. O uso da estereoscopia para a interpretação das fotos permitiu estudar o relevo a partir de uma visão tridimensional, com as pequenas feições do relevo e as marcas dos processos morfodinâmicos sendo facilmente identificadas, enquanto as medidas de extensão e ângulos de inclinação das vertentes puderam ser determinadas com maior nível de precisão.
\end{abstract}

Dentre as várias possibilidades de material fornecido por essa tecnologia estão a aerofotogrametria, fotointerpretação e processamento de imagens de radar e satélite (SILVA \& SANTOS op. cit.). Parte dessas informações são de fácil acesso, assim como alguns softwares onde elas são manipuladas, por outro lado alguns dados de satélite possuem um valor comercial pouco viável para alguns pesquisadores, que devido a essa dificuldade elaboram técnicas de melhoramento das imagens acessíveis como a ampliação linear de contraste, o uso de operações aritméticas, a transformação por IHS, a geração de composições coloridas, dentre tantas outras que facilitam a interpretação das imagens (FLORENZANO, 2011).

Uma das razões para esta disparidade é a qualidade na resolução das imagens que dependem de uma série de fatores dentre eles o sistema de varredura dos sensores, faixa espectral, tempo de revisita (MOREIRA, 2012).

A aplicação do sensoriamento remoto aliado ao conhecimento já produzido sobre a dinâmica da paisagem permite uma melhor análise do relevo e sua estrutura, contribuindo assim com a otimização da pesquisa, reduzindo tempo e custos em campo e propiciando uma melhor visualização, em diferentes escalas, dos compartimentos morfológicos.

Novo (1992) citando o trabalho de Verstappen (1977) afirma que segundo esse autor há três aplicações do sensoriamento remoto na Geomorfologia: 1) aplicações cartográficas; 2) aplicações topográficas; e 3) interpretação geomorfológica completa, esta entendida não se restringindo apenas aos aspectos visíveis, mas também as inferências de processos. Assim, pode-se afirmar que o uso das técnicas em sensoriamento remoto aos estudos do relevo torna possível a classificação os domínios dos aspectos morfoestruturais auxiliando no mapeamento geomorfológico e possibilita o entendimento da morfogênese das feições da superfície terrestre (IBGE, 2009).

Nesta perspectiva, a presente pesquisa busca mostrar como os pesquisadores têm empregado essa técnica na geomorfologia na atualidade, através de análise e classificações organizadas em linhas de pesquisa e objetivos dentro da geomorfologia dos trabalhos submetidos nos eventos 
científicos mais importantes nas áreas de sensoriamento remoto e geomorfologia. Além disso, mostrar e discutir a diferença quantitativa de produção entre as regiões do país. Essas técnicas também possibilitam os estudos voltados para a morfodinâmica visto que é possível inferir sobre as causas e a extensão dos processos

Desse modo, escolheram-se dois dos principais eventos nacionais para a realização deste estudo nas suas duas últimas edições, foram eles: o Simpósio Brasileiro de Sensoriamento Remoto por se tratar de um evento acerca do tema central deste estudo e possuir eixo específico para os estudos geomorfológicos. E o Simpósio Nacional de Geomorfologia que abrange todas as vertentes da geomorfologia.

\section{METODOLOGIA}

Devido à elevada amplitude das aplicações em sensoriamento remoto nos estudos geomorfológicos, limitou-se o escopo desta análise àquelas direcionadas ao estudo dentro das grandes linhas geomorfológicas fluvial, costeira, estrutural e dinâmica/climática. (CHRISTOFOLETTI, 1980; GUERRA \& CUNHA, 2009).

Nesse sentido foram analisados os trabalhos publicados nos anais dos eventos nacionais que tratam dessas temáticas, entre os anos de 2015 a 2018. Como fontes de dados foram consultados os anais dos XVII e XVIII Simpósio Brasileiro de Sensoriamento Remoto - SBSR, respectivamente ocorridos em 2015 (João Pessoa, PB) e 2017 (Santos, SP); e os anais do XI e XII Simpósio Nacional de Geomorfologia - SINAGEO, respectivamente realizados em 2016 (Maringá, PR) e 2018 (Crato, CE).

A fim de obter um panorama do estado da arte, ou seja, do grau de desenvolvimento dos estudos em sensoriamento remoto aplicado à geomorfologia, optou-se por selecionar os eixos temáticos de cada simpósio que estão relacionados com as quatros grandes linhas da geomorfologia supracitadas.

Posteriormente, os trabalhos foram separados por objetivos: (a) identificação/caracterização; (b) classificação/mapeamento; (c) avaliação/inventário; e (d) monitoramento/manejo. Na sequência, procurou-se definir o local onde os estudos foram realizados por regiões, o tipo de imagem utilizada e sua resolução, os softwares utilizados nas análises, a finalidade e o objetivo principal do estudo, os principais produtos e métodos de análise de dados.

Por fim foi feita uma espacialização das produções que contribuiu numa discussão a respeito das disparidades produtivas entre as regiões.

\section{RESULTADOS E DISCUSSÃO}

\section{Publicações analisadas por linhas geomorfológicas}

Foram encontrados na pesquisa nos anais dos eventos 34 trabalhos relacionados com o uso do sensoriamento remoto para os estudos geomorfológicos, sendo que mais da metade desses trabalhos foram realizados dentro da linha da geomorfologia fluvial. Em segundo lugar ficaram os trabalhos relacionados ao estudo dentro da geomorfologia dinâmica, com 7 trabalhos (Figura 1).

Dos 19 trabalhos da linha de geomorfologia fluvial, 9 estão relacionados a estudos realizados em bacias hidrográficas, os outros trabalhos abordam questões ligadas a barras fluviais (QUEIROZ et al., 2016), planície inundação (REIS et al., 2017), morfologia de ilhas (SOUZA \& RODRIGUES, 2017) e transporte de sedimentos (CREMON et al, 2018).

Já os trabalhos de geomorfologia dinâmica estão em sua maioria relacionados com os estudos de movimentos de massas em encostas (AMARAL et al., 2016; QUINA et al., 2018), cabe 
destacar nessa linha o uso de imagens de sensor com boa qualidade de resolução espacial, como as do sensores Ikonos, RapidEye, CBERS, Palsar e imagens de Veículo Aéreo Não Tripulado (VANT).

Os três trabalhos encontrados da linha estrutural são realizados com vistas na análise do relevo a partir a extração de lineamento morfoestruturais (LIMA et al., 2015; ALVES et al., 2017). Por fim, os trabalhos realizados na linha costeira são relacionados com linha de costa, dunas e erosão costeira, como os trabalhos realizados por Silva et al. (2015), Lael et al. (2016) e Biffi \& Souza Filho (2018).

Figura 1. Quantidade de trabalhos anais das edições XVII e XVIII do SBSR e XI e XII do SINAGEO por linhas da geomorfologia.

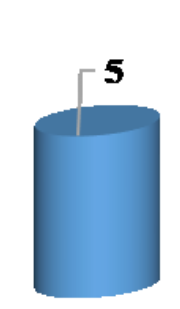

Costeira

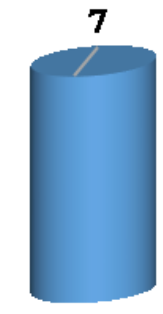

Dinâmica

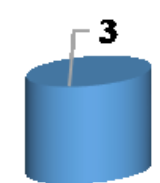

Estrutural

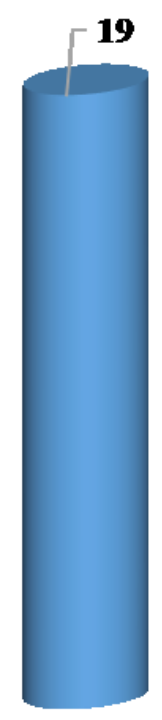

Fluvial

Elaboração: AUTORES, 2018

\section{Publicações analisadas por objetivos}

Os objetivos dos trabalhos foram divididos em 4 categorias (Figura 2), sendo a primeira delas denominada Identificação/caracterização, está teve a menor participação apenas no SBSR 2015, a categoria está bem distribuída nas linhas de pesquisa, os trabalhos buscam identificar feições morfométricas de bacias, identificação de cicatrizes de deslizamento, lineamentos estruturais, áreas inundáveis. Em geral os trabalhos apresentam uma produção de dados primários que servirão de base para trabalhos posteriores. A segunda categoria é a de Classificação/mapeamento, estando presente em todos os eventos, traz trabalhos de mapeamento de feições morfológicas, delimitação de bacias hidrográficas, e aplicação de índices. Os trabalhos se caracterizam como secundários, ou seja, que utilizaram dados primários já produzidos anteriormente. Os trabalhos desta categoria estão presentes apenas na linha de Geomorfologia Fluvial.

A terceira categoria é a de Avaliação/Inventário, está assim como as anteriores também está presente em todos os eventos, porém não aparece na linha de geomorfologia costeira, são trabalhos que buscam em maioria a aplicação de índices para explicar fenômenos de 
deslizamento e alta carga de sedimentos em suspensão nos rios, os trabalhos também apresentam técnicas e comparam dados já existentes buscando um aperfeiçoamentos dos trabalhos.

Monitoramento/manejo é a quarta e última categoria, está presente nos eventos de 2015, 2017 e 2018, os seus trabalhos são voltados às linhas de Geomorfologia fluvial e Costeira, são pesquisas que objetivam ter conhecimento de processos superficiais e subsuperficiais que ocorrem em áreas que tem uma dinâmica rapidamente alterada como barras, ilhas e costas.

Figura 2. Quantidade de trabalhos publicados nos anais das edições XVII e XVIII do SBSR e XI e XII do SINAGEO por objetivo

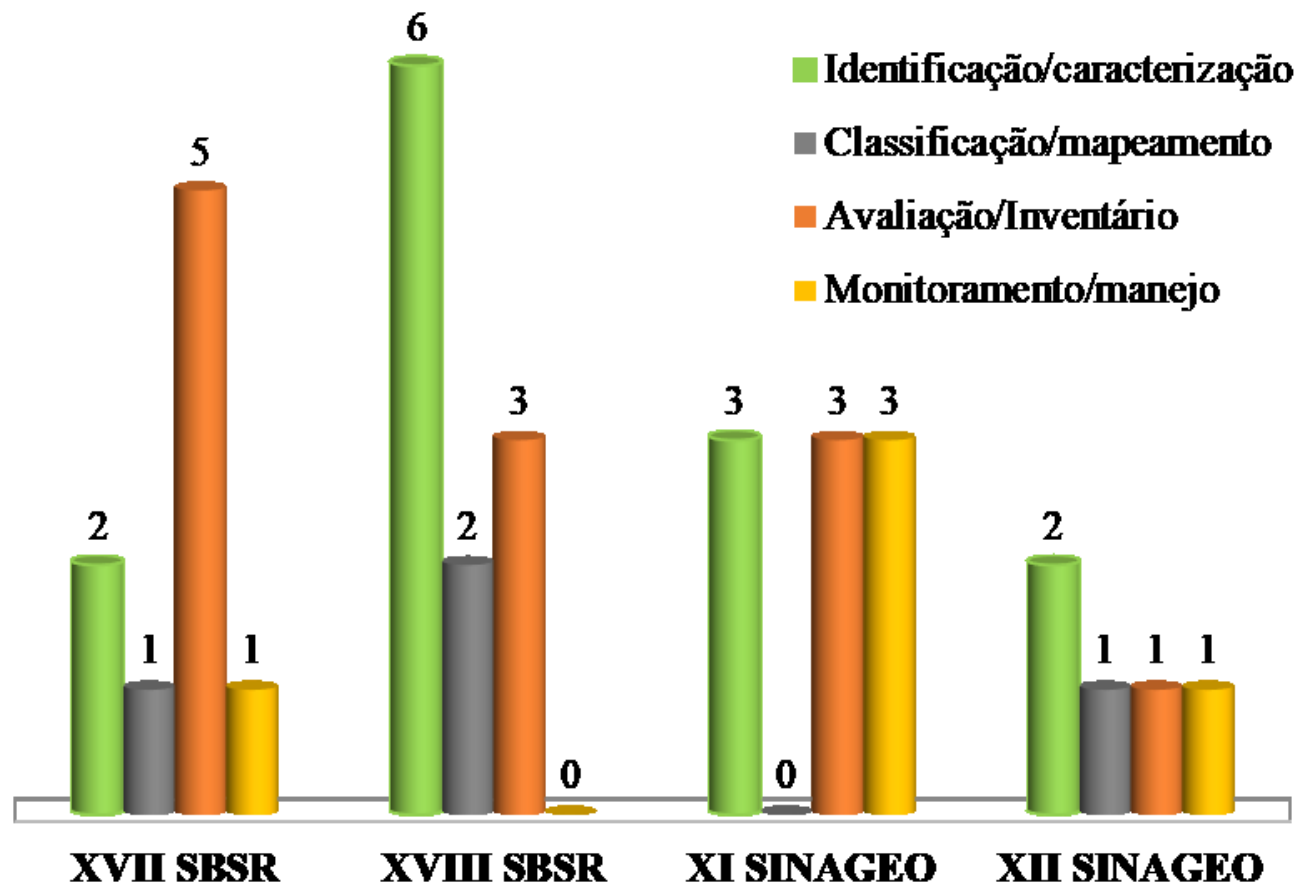

Elaboração: AUTORES, 2018.

\section{Distribuição espacial da produção}

Para compreender a distribuição dos trabalhos realizados no Brasil, foram feitos mapas temáticos (Figura 3) para espacializar os estudos publicados nos anais dos eventos realizados e assim tentar elaborar ideias que explique a maior ou menor produção no país ao longo dos eventos.

No caso do XVII SBSR, foram publicados 10 trabalhos que correspondem à temática analisada nessa pesquisa, sendo 6 deles produzidos na região Nordeste e destes, sendo metade desses realizados na Bacia Paraíba no próprio estado da Paraíba (ALVES et al., 2015; ANDRADE FILHOS et al., 2015; LIMA et al., 2015), o que pode pressupor que a localidade do evento foi um dos fatores para este resultado. Assim como aconteceu no SINAGEO de 2016, o evento foi promovido na cidade de Maringá - PR e teve um total de 8 trabalho de sensoriamento remoto aplicado à geomorfologia, sendo 6 deles na região Sul e destes, 2 no estado onde aconteceu o evento (BIFFI \& SOUZA FILHO, 2016; MACHADO \& CAMARGO FILHO, 2016). 
Figura 3. Espacialização dos trabalhos publicados nos anais dos eventos do Simpósio Brasileiro de Sensoriamento Remoto e Simpósio Nacional de Geomorfologia, entre 2015 e 2018

TRABALHOS DO SBSR 2015

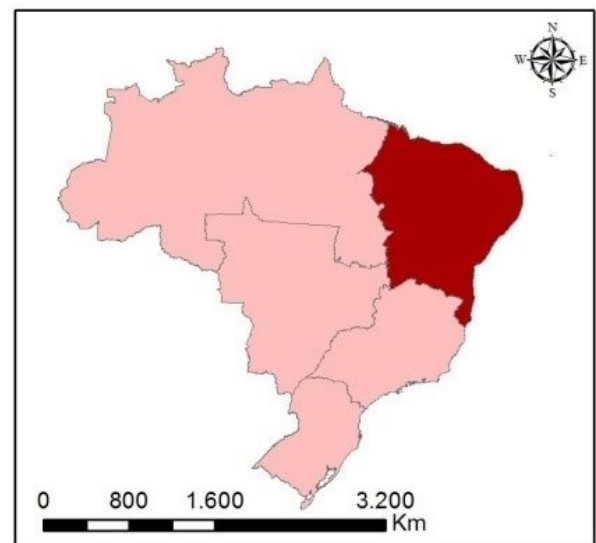

Norte (1)

Sul (1)

Nordeste (6)

Sudeste (1)

Centro-oeste (1)

TRABALHOS SINAGEO 2016

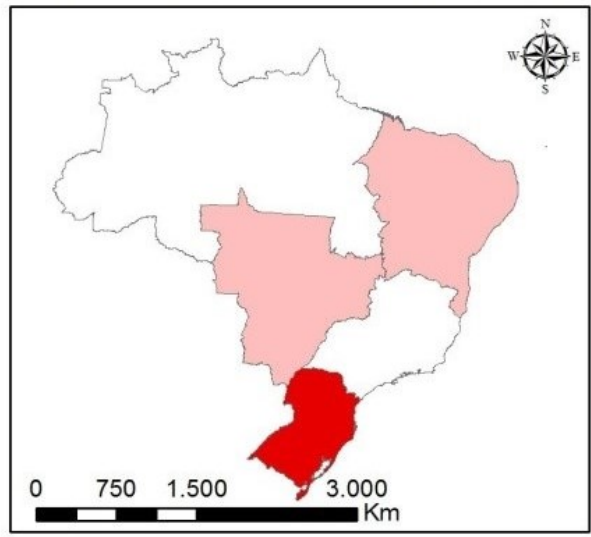

Norte (0)

Sul (6)

Nordeste (1)

Sudeste (0)

Centro-oeste (1)
TRABALHOS DO SBSR 2017

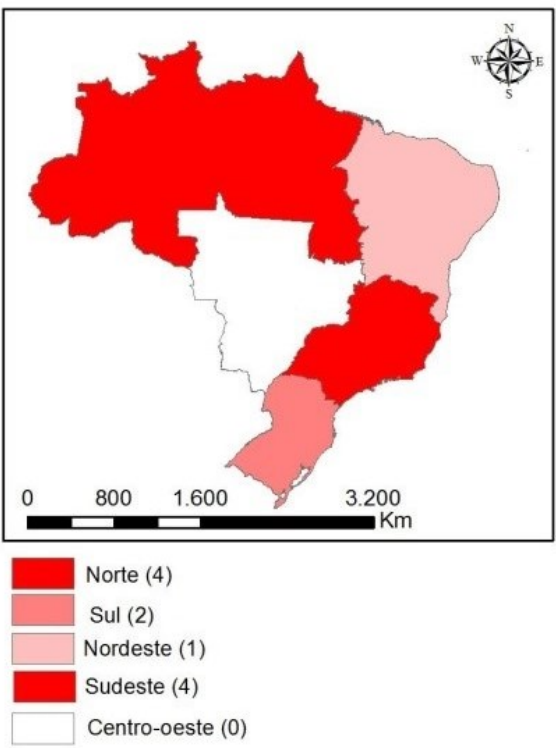

TRABALHOS SINAGEO 2018

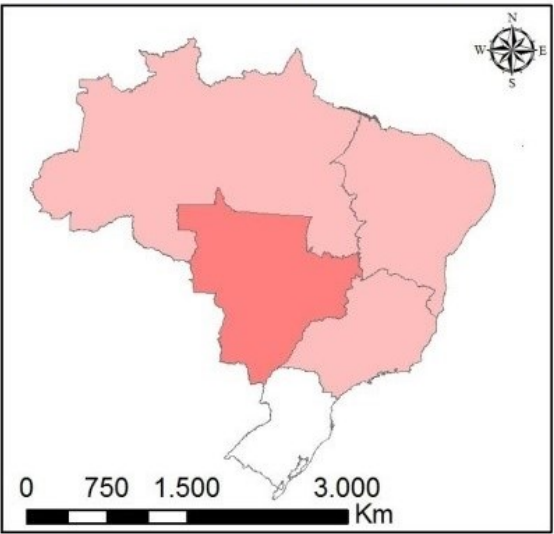

Norte (1)

Sul (0)

Nordeste (1)

Sudeste (1)

Centro-oeste (2)

Elaboração: AUTORES, 2018.

Já o SBSR XVIII teve uma produção um pouco maior que evento anterior, com 11 trabalhos, sendo 4 deles da região Sudeste, destes, 2 deles do Espírito Santo. Porém, diferente do SBSR 2015, este não teve uma grande diferença entre as regiões, pois houve quatro trabalhos realizados na região Norte e o Sul duas, a região Centro-oeste ficou sem representação neste evento. O SINAGEO de 2018 aconteceu na cidade do Crato - CE com menor produção se comparado ao anterior, com 5 trabalhos sendo dois deles produzidos no Centro-oeste e o restante dividido entre as regiões Norte, Nordeste e Sudeste, no caso do SINAGEO 2018, foi a região Sul que ficou sem representação. 


\section{Outras características relevantes em relação as publicações}

No que diz respeito a escala de analise espacial dos trabalhos, as diferenças ficaram bem visíveis sobretudo no tipo de dado utilizado. Nos trabalhos realizados em macro escala (SIRANGELO, 2015; FILHO et al, 2015; ALVES et al, 2017), foram utilizados dados Topodata e SRTM com resoluções espaciais de 30 e 90 metros, que são gratuitas e de fácil acesso, fato que corrobora com a opinião de Costa \& Silva (2015) quando afirmam que esses produtos podem ser considerados razoáveis em escalas pequenas a médias, mas não é aconselhado para escalas de estudos detalhados.

Entretanto, outros trabalhos tiveram a necessidade de uma escala de detalhe e semidetalhe (DUARTE et al, 2017; SILVA \& NISHIYAMA, 2016) como os de análise de sedimentos em suspensão, neles seus autores utilizaram os dados provenientes do RapidEye, este fornece imagens com resolução de $5 \mathrm{~m}$ o que possibilita esse tipo de trabalho, assim como feições erosivas de pequena escala, que também foi resultado de alguns dos trabalhos que utilizaram este tipo de dado. Isso decorre da necessidade de um maior detalhe que esses estudos geodinâmicos necessitam, sendo essas imagens de alta resolução responsáveis pelo reconhecimento das feições de detalhes e processos morfodinâmicos atuantes (IBGE, 2009). Em outros trabalhos que também necessita de detalhes os autores utilizaram imagens do CBERS-2, dos VANTs e do Ikonos, que possuem resolução de até 2,7m como no caso do CBERS-2 para identificar feições em barras de canal. A maioria dessas imagens com alta resolução espacial tem um valor comercial agregado, o que pode explicar o baixo número de trabalhos realizados com esses dados.

Outros dados também foram utilizados como os provenientes do satélite ALOS do sensor Palsar e Google Earth para trabalhos que assim com os primeiros citados também não precisaram de uma escala de detalhe. Em geral, constatou-se que a maioria dos trabalhos encontrados teve o processamento digital de imagens realizados pelos softwares proprietários e gratuitos como o Arcgis, Envi, Global Mapper, Spring e Qgis.

Cabe destacar que as metodologias utilizadas no processamento digital de imagens para a obtenção das informações nos trabalhos encontrados foram em sua maioria ligadas a composição de bandas multiespectrais, filtragem espacial, classificação supervisionada, fotointerpretação e, destaca-se na linha de geomorfologia fluvial, a extração de parâmetros morfométricos.

Em relação aos resultados obtidos nos trabalhos todos demonstraram-se satisfatórios para os objetivos propostos, evidenciando a grande contribuição que as técnicas de sensoriamento remotos tem aos estudos voltados para a análise da superfície da Terra bem como os processos ocorridos na mesma.

\section{CONSIDERAÇÕES FINAIS}

A utilização do sensoriamento remoto nos estudos geomorfológicos está sempre presente nos eventos científicos de maior representatividade nacional da área, no caso do SBSR houve uma quantidade maior de trabalhos apresentados do que no SINAGEO, isso porque neste último o conjunto de geotecnologias utilizadas é mais amplo e nem sempre se utiliza somente técnicas de sensoriamento remoto, integrando-as aos Sistemas de Informação Geográficas.

A Geomorfologia é um ramo da Geografia que tem como objeto de estudo a superfície e subsuperfície da Terra, e sendo ela tão diversificada foi necessário ao longo dos estudos geomorfológicos dividir esse ramo tão importante por contexto e processos. No caso deste 
trabalho, foi dividida em geomorfologia costeira, fluvial, dinâmica e estrutural. Cada uma desta avalia o relevo sob um grande aspecto condicionante da paisagem, como a ação marinha no caso da costeira, as drenagens na fluvial, o clima na dinâmica e a estrutura geológicas na estrutural, cada linha dessa precisa de condições de avaliação específicas e por isso dependem de tipos de dados diferentes. Nesta pesquisa, os trabalhos avaliados mostraram que o sensoriamento remoto atende a estas necessidades e contribui na otimização do tempo e do resultado nas pesquisas.

A partir da análise das temáticas dos trabalhos percebe-se o alto grau de instrução dos pesquisadores que utilizam as técnicas de sensoriamento aplicadas aos estudos geomorfológicos, desde estudos em macroescalas até escalas de semidetalhe. Foram utilizadas em maioria imagens gratuitas e de fácil acesso, porém alguns trabalhos teve a necessidade do uso de dados com representativo valor econômico, como os dados do IKONOS e dos VANTs. Quanto aos softwares, a maioria dos autores utilizara apenas um programa para a realização de todo o trabalho, o que mostra que estes estão cada vez mais eficientes e atendem na maioria das vezes as necessidades do pesquisador que ao longo do tempo vem se aperfeiçoando e hoje a rede está cheia de tutoriais e aulas a respeito do uso da tecnologia abordada nesta pesquisa.

No que diz respeito aos locais onde estão sendo feitos os trabalhos, eles estão bem distribuídos entre as regiões, porém alguns aspectos como a localidade dos eventos atrapalham a apresentação destes, visto que o Brasil é um país bastante amplo, surgem daí as dificuldades de locomoção dos pesquisadores que em maioria são estudantes de graduação de pós-graduação.

\section{REFERÊNCIAS}

ALVES, F. C. et al. Identificação semiautomática de lineamentos morfoestruturais no norte da Bacia Paraíba, PB. In: SIMPÓSIO BRASILEIRO DE SENSORIAMENTO REMOTO, 18. (SBSR), 2017, Santos. Anais... São José dos Campos: INPE, 2017. p. 1084-1091.

ALVES, F. C.; ROSSETI, D. F.; VALERIANO, M. M. Identificação de subambientes de delta a partir de imagem PALSAR/ALOS-1. In: SIMPÓSIO BRASILEIRO DE SENSORIAMENTO REMOTO, 17. (SBSR), 2015, João Pessoa. Anais... João Pessoa: INPE, 2015. p. 903-910.

AMARAL, L. L. et al. Proposta metodológica para avaliação de produção de sedimentos em feições erosivas a partir de MDS de alta resolução. In: SIMPÓSIO NACIONAL DE GEOMORFOLOGIA, 11. (SINAGEO), 2016, Maringá. Anais... Maringá: UGB, 2016. Disponível em: http://www.sinageo.org.br/2016/trabalhos/6/6-551-1251.html. Acesso em: 14 out. 2018.

ANDRADES FILHO, C. O; ROSSETI, D. F.; BEZERRA, F. H. R. Geomorfometria e aeromagnetometria integradas para análise da correspondência entre lineamentos morfoestruturais e magnéticos na Bacia Paraíba, nordeste do Brasil. In: SIMPÓSIO BRASILEIRO DE SENSORIAMENTO REMOTO, 17. (SBSR), 2015, João Pessoa. Anais... João Pessoa: INPE, 2015. p. 3384-3391.

BIFFI, V. H. R.; SOUZA FILHO, E. E. A. Quantificação da erosão marginal no arquipélago Mutum Porto Rico entre 1985 e 2009 por meio de imagens Lansat 5 - TM. In: SIMPÓSIO NACIONAL DE GEOMORFOLOGIA, 11. (SINAGEO), 2016, Maringá. Anais... Maringá: UGB, 2016. Disponível em: http://www.sinageo.org.br/2016/trabalhos/3/3-248-1495.html. Acesso em: 14 out. 2018.

CORRÊA, A. C. B. A geografia física: uma pequena revisão de seus enfoques. RIOS - Revista da FASETE, Paulo Afonso, BA, v. 1, p. 170-180, 2005.

COSTA, R. O; SILVA, T. M CONTRIBUIÇÕES DAS TÉCNICAS DE SENSORIAMENTO REMOTO PARA MAPEAMENTOS GEOMORFOLÓGICOS. In: SIMPÓSIO BRASILEIRO DE GEOGRAFIAA FÍSICA APLICADO. (SBGFA), 2015. Anais... Teresina-PI: 2015.

CREMON, E. H.; SILVA, A. M. S.; MONTANHER, O. C. Estimativa de sedimentos suspensos por reflectância aparente dos dados TM/Landsat-5 no Rio Araguaia. In: SIMPÓSIO NACIONAL DE 
GEOMORFOLOGIA, 12. (SINAGEO), 2018, Crato. Anais... Crato: UGB, 2018. Disponível em: http://www.sinageo.org.br/2018/trabalhos/9/9-362-165.html. Acesso em: 14 out. 2018.

DUARTE, C. R. et al. Dispersão de sedimentos e morfologia subsuperficial: avaliação a partir de imagens RapidEye na área de entorno do Terminal Portuário do Pecém, Ceará. In: SIMPÓSIO BRASILEIRO DE SENSORIAMENTO REMOTO, 18. (SBSR), 2017. Anais... Santos-SP: INPE, 2017. p. 5124-5131.

INSTITUTO BRASILEIRO DE GEOGRAFIA E ESTATÍSTICA - IBGE. Manual técnico de geomorfologia. 2 ed. Rio de Janeiro: 2009. 182 p.

FILHO, C. O. A. et al. Geomorfometria e aeromagnetometria integradas para análise da correspondência entre lineamentos morfoestruturais e magnéticos na Bacia Paraíba, nordeste do Brasil. In: SIMPÓSIO BRASILEIRO DE SENSORIAMENTO REMOTO, 18. (SBSR), 2015, Santos. Anais... João Pessoa-PB: INPE, 2015. p. 3384-3391.

LEAL, K. B.; OLIVEIRA, U. S.; ESPINOZA, J. M. A. Análise temporal da variação da linha de costa para os balneários da Praia do Mar Grosso, São José do Norte - RS a partir do uso de geotecnologias. In: SIMPÓSIO NACIONAL DE GEOMORFOLOGIA, 11. (SINAGEO), 2016, Maringá. Anais... Maringá: UGB, 2016. Disponível em: http://www.sinageo.org.br/2016/trabalhos/6/6-161-1635.html. Acesso em: 14 out. 2018.

LIMA, J. C. F. Análise morfoestrutural da Bacia Paraíba com radar interferométrico. In: SIMPÓSIO BRASILEIRO DE SENSORIAMENTO REMOTO, 17. (SBSR), 2015, João Pessoa. Anais... João Pessoa: INPE, 2015. p. 567-573.

LIMA, J. C. F. et al. Análise morfoestrutural da Bacia Paraíba com radar interferométrico. In: SIMPÓSIO BRASILEIRO DE SENSORIAMENTO REMOTO, 17. (SBSR), 2015, João Pessoa. Anais... João Pessoa: INPE, 2015. p. 567-573.

MACHADO, F. E; CAMARGO FILHO, M. Identificação de áreas úmidas por meio da missão CBERS-2 na região Sul do município de Guarapurava-PR. In: SIMPÓSIO NACIONAL DE GEOMORFOLOGIA, 11. (SINAGEO), 2016, Maringá. Anais... Maringá: UGB, 2016. Disponível em: http://www.sinageo.org.br/2016/trabalhos/6/6-397-91.html. Acesso em: 14 out. 2018.

NOVO, E. M. L. de M. Sensoriamento remoto: princípios e aplicações. 2. ed. São Paulo: Edgard Blücher, 1992. 308 p.

QUEIROZ, P. H. B. et al. Evolução morfológica de barras fluviais no canal do rio Jaguaribe a jusante da barragem do Açude Castanhão-CE. In: SIMPÓSIO NACIONAL DE GEOMORFOLOGIA, 11. (SINAGEO), 2016, Maringá. Anais... Maringá: UGB, 2016. Disponível em: http://www.sinageo.org.br/2016/trabalhos/3/3-178-976.html. Acesso em: 14 out. 2018.

QUINTA, R. R.; COSTA, R. M.; ZAIDAN, R. T. Mapeamento de estabilidade de encostas com modelo SINMAP, a partir da comparação dos sensores Lidar e Alos Palsar: uma aplicação na bacia hidrográfica do Córrego Tapera, Juiz de Fora-MG. In: SIMPÓSIO NACIONAL DE GEOMORFOLOGIA, 12. (SINAGEO), 2018, Crato. Anais... Crato: UGB, 2018. Disponível em: http://www.sinageo.org.br/2018/trabalhos/9/9-220-1936.html. Acesso em: 14 out. 2018.

REIS, J. B. C.; BERTONCINI, A. L. S.; RENNÓ, C. D. Mapeamento da planície de inundação do rio Sapucaí para a área urbana do município de Itajubá (MG). In: SIMPÓSIO BRASILEIRO DE SENSORIAMENTO REMOTO, 18. (SBSR), 2017, Santos. Anais... São José dos Campos: INPE, 2017. p. 3719-3726.

Silva, G.A. et al. Mapeamento dos processos erosivos na bacia hidrográfica do Ribeirão São Lourenço em Ituiutaba, Minas Gerais: SIMPÓSIO NACIONAL DE GEOMORFOLOGIA. (SINAGEO), 2016. Anais... Maringá-PR: 2016.

SILVA, F. E; AMARO, V. E; MATO, M. F.. Análise multitemporal do campos de dunas móveis do litoral setentrional do Rio Grande do Norte. In: SIMPÓSIO BRASILEIRO DE SENSORIAMENTO REMOTO, 17. (SBSR), 2015, João Pessoa. Anais... João Pessoa: INPE, 2015. p. 6966-6973.

SILVA, J. M. F.; SANTO, L. J. C. O sensorimentos remoto na pesquisa geomorfológica: aplicação no município de Antonina, Paraná. Revista Geonorte, Edição especial, V.2, N, 4, p.13636-1645, 2012.

SOUZA, S. V.; RODRIGUES, S. W. P. Reconhecimento e mapeamento das feições fluviais da "Ilha Grande do Tapará" (Santarém-PA) a partir do processamento de imagem RapidEye e SAR-SIPAM. In: 
SIMPÓSIO BRASILEIRO DE SENSORIAMENTO REMOTO, 18. (SBSR), 2017, Santos. Anais... São José dos Campos: INPE, 2017. p. 4843-4850.

SIRANGELO, F. R. et al. Análise morfométrica das sub-bacias da bacia hidrográfica do TaquariAntas/RS, Brasil. In: SIMPÓSIO BRASILEIRO DE SENSORIAMENTO REMOTO, 18. (SBSR), 2015, Santos. Anais... João Pessoa-PB: INPE, 2015. p. 4930-7937. 